\title{
Post-selected weak measurement beyond the weak value
}

\author{
Tamás Geszt:* \\ Department of Physics of Complex Systems, Eötvös University, Budapest, Hungary
}

\begin{abstract}
Closed expressions are derived for the quantum measurement statistics of pre-and postselected gaussian particle beams. The weakness of the pre-selection step is shown to compete with the non-orthogonality of post-selection in a transparent way. The approach is shown to be useful in analyzing post-selection-based signal amplification, allowing measurements to be extended far beyond the range of validity of the well-known Aharonov-Albert-Vaidman limit.

PACS numbers: 03.65.Ta, 42.50.Xa, 03.65.Yz
\end{abstract}

Pre-and postselected weak measurements [1], with their surprising mathematical properties, often traced back to a weird combination of Fourier components [2], have raised considerable interest, strongly supported by experimentally accessible predictions [3, 4]. The standard discussion is centered about the so-called weak value

$$
\langle\hat{A}\rangle_{\text {weak }}=\frac{\langle f|\hat{A}| i\rangle}{\langle f \mid i\rangle}
$$

of an observable $\hat{A},|i\rangle$ and $|f\rangle$ being two vectors - an initial and a final ("post-selected") state - in the Hilbert space on which $\hat{A}$ - say, a spin component of an atom - acts. Remaining with that example, the AharonovAlbert-Vaidman (AAV) procedure is a sophisticated twostep Stern-Gerlach-type measurement of that spin component. The first Stern-Gerlach separation, coupling the spin by magnetic interaction to the transverse position $z$ of the atomic beam, is weak, i.e. the resulting shifts $\pm d$ of the two beams are much less than the transverse width $w$ of each beam. That first step is followed by an appropriately oriented second - strong - Stern-Gerlach separation, marking out one of the branches for further processing - that is called the post-selection. Finally [5], the measured value of $\hat{A}$ is read off through the statistics of atoms detected within the postselected branch at different displacements $z[6]$. The full sequence can result in detecting displacements on the average much larger than $d$, being proportional to the real part of the weak value, Eq. (11). A feasible alternative implementation of the scheme is to use light beams, polarization taking the place of spin, and birefringence - linear or circular - being used for the Stern-Gerlach-type separation. Most recently, several experiments [7, 8] used the scheme to measure weak birefringence itself, giving rise to subnanometer-scale displacements; the AAV post-selection served for signal amplification of that [9]. There was one change with respect to the scheme outlined above: the new measurements exploit the possibility of measuring the imaginary part of the weak value, which transforms the displacements into momentum space, with strongly enhanced amplification.

*Electronic address: geszti@elte.hu
The aim of the present note is to analyze the situation in a straightforward manner, as a two-beam interference phenomenon in which visible contrast is created by the AAV sequence of weak pre-selection and strong post-selection. The procedure can be analyzed in full generality; for simplicity, we present the relevant formulas for gaussian beams. In that context the AAV weak value, Eq. (11), appears as a complex expression furnishing measurable mean values in a well-defined limiting case. With that knowledge, practical tools are obtained to explore and analyze a wide range of experimentally accessible data far beyond that limiting case.

In the analysis that follows we find that although for $d / w \rightarrow 0$ the measured mean value approaches the AAV weak value Eq. (1), a small but finite weakness ratio $d / w$ is competing in smallness with the distance from full ortogonality of the pre- and postselected states. We derive a closed expression for the measured mean value, valid for any degree of pre-selection weakness and postselection non-orthogonality. Under the single condition of weakness, $d / w \ll 1$, the formula is reduced to a simple non-linear function of $d / w$, which if used for data fitting, can result in strongly enhanced signal amplification.

For the sake of concreteness, we speak of spin-1/2 particles prepared in a definite initial spin state $\left(\begin{array}{l}\alpha \\ \beta\end{array}\right)$, and the observable one wants to measure is the spin component $\hat{\sigma}_{z}$. The corresponding Stern-Gerlach separation, followed by free flight until reaching the detectors, produces the respective displacements $\pm d$ in the $z$ direction for the two spin components. We assume that the particles are propagating along the $y$ direction in gaussian beams, the width of which reaches the value $w$ in the plane of the detectors. Weak separation/measurement is defined by the condition $d \ll w$, i.e. the two beams remain strongly overlapping. Omitting trivial events in the other space dimensions, this first step of weak SternGerlach separation can be described as

$$
|i\rangle=\Phi(z)\left(\begin{array}{l}
\alpha \\
\beta
\end{array}\right) \longrightarrow\left(\begin{array}{l}
\alpha \Phi(z-d) \\
\beta \Phi(z+d)
\end{array}\right),
$$

where $\Phi(z)=\exp \left(-z^{2} / 4 w^{2}\right) / \sqrt{w \sqrt{2 \pi}}$ is the normalized transverse wave function of the beam in the direction $z$ of separation. Spin-orbital entanglement associates the two beams to orthogonal spin states, therefore straight- 
forward detection would just add the intensities, to produce the probability distribution function

$$
p(z)=|\alpha|^{2} \Phi^{2}(z-d)+|\beta|^{2} \Phi^{2}(z+d)
$$

and the corresponding mean displacement

$$
\langle z\rangle=\left(|\alpha|^{2}-|\beta|^{2}\right) d
$$

which is practically invisible above the gaussian background of width $w$.

However, the spin degree of freedom offers the possibility to create phase contrast by rotating the spin basis, which is subsequently exploited in the post-selection procedure (see below). The experimentally more accessible case of photons with two orthogonal states of polarization, and their spatial separation by one of the many effects giving rise to birefringence, then post-selecting by polarization rotation, makes no difference in the description, only the physics behind the displacement $d$ and the method of preparation of pre- and postselected states change.

In the example at hand the post-selection step, to be carried out before detection, consists in a second, this time strong Stern-Gerlach separation in a different direction $\xi$, rotated with respect to the initial one, fully separating the above state vector into two non-overlapping beams of the corresponding orthogonal spin states $\left(\begin{array}{l}\gamma \\ \delta\end{array}\right)$

$$
\begin{aligned}
& \text { and }\left(\begin{array}{c}
\delta^{*} \\
-\gamma^{*}
\end{array}\right): \\
& \qquad f_{1}(\xi)\left(\alpha \gamma^{*} \Phi(z-d)+\beta \delta^{*} \Phi(z+d)\right)\left(\begin{array}{l}
\gamma \\
\delta
\end{array}\right) \\
& \quad+f_{2}(\xi)(\alpha \delta \Phi(z-d)-\beta \gamma \Phi(z+d))\left(\begin{array}{c}
\delta^{*} \\
-\gamma^{*}
\end{array}\right),
\end{aligned}
$$

where $f_{1}(\xi)$ and $f_{2}(\xi)$ are two non-overlapping functions of the post-selection coordinate $\xi$.

It is this step which creates visible phase contrast between the two $z$-shifted peaks, in each of the postselected components. Any of them can be projected out by placing the detector into the corresponding band; e.g. for the first component: where $f_{1}(\xi) \neq 0 ; f_{2}(\xi)=0$. The final result is obtained by scanning with the detector along $z$, while keeping $\xi$ unchanged. That is the final, non-unitary quantum measurement, the statistics of which is expected to furnish as output, through Born's rule, the fraction of incoming particles detected in the postselected channel, at displacement $z$ :

$$
\begin{aligned}
& q(z):=\left|\alpha \gamma^{*} \Phi(z-d)+\beta \delta^{*} \Phi(z+d)\right|^{2} \\
& =|\alpha|^{2}|\gamma|^{2} \Phi^{2}(z-d)+|\beta|^{2}|\delta|^{2} \Phi^{2}(z+d) \\
& \quad+2 \Re\left(\alpha \beta^{*} \gamma^{*} \delta\right) e^{-\frac{d^{2}}{2 w^{2}}} \Phi^{2}(z)
\end{aligned}
$$

where the last term is the contribution of visible interference, created in the process of post-selection.
The analogous expression for the complementary channel, $f_{2}(\xi) \neq 0 ; f_{1}(\xi)=0$, reads $|\alpha|^{2}|\delta|^{2} \Phi^{2}(z-d)+$ $|\beta|^{2}|\gamma|^{2} \Phi^{2}(z+d)-2 \Re\left(\alpha \beta^{*} \gamma^{*} \delta\right) e^{-\frac{d^{2}}{2 w^{2}}} \Phi^{2}(z)$. Adding that to Eq. (6), one obtains the full preselected distribution function, Eq. (3). This result [10] confirms that the procedure is a "true" post-selection in the Bayesian sense, using the channel information to cut the slightly asymmetric preselected distribution into two strongly asymmetric postselected ones, which is the basis of signal amplification [11, 12].

Normalizing Eq. (6) to the postselected fraction of the incoming particles, we obtain the postselected probability distribution function

$$
p_{\text {post }}(z)=\frac{q(z)}{|\alpha|^{2}|\gamma|^{2}+|\beta|^{2}|\delta|^{2}+2 \Re\left(\alpha \beta^{*} \gamma^{*} \delta\right) e^{-\frac{d^{2}}{2 w^{2}}}},
$$

where the denominator is the fraction of all particles postselected into spin state $\left(\begin{array}{l}\gamma \\ \delta\end{array}\right)$, irrespective of their displacement $z$.

From Eqs. (6) and (7) one immediately reads the postselected mean displacement

$$
\begin{aligned}
\langle z\rangle_{\text {post }} & =\frac{|\alpha|^{2}|\gamma|^{2}-|\beta|^{2}|\delta|^{2}}{|\alpha|^{2}|\gamma|^{2}+|\beta|^{2}|\delta|^{2}+2 \Re\left(\alpha \beta^{*} \gamma^{*} \delta\right) e^{-\frac{d^{2}}{2 w^{2}}}} d \\
& \approx \frac{\left(|\alpha|^{2}|\gamma|^{2}-|\beta|^{2}|\delta|^{2}\right)(d / w)}{\left|\alpha \gamma^{*}+\beta \delta^{*}\right|^{2}-\Re\left(\alpha \beta^{*} \gamma^{*} \delta\right)(d / w)^{2}} w
\end{aligned}
$$

In the last step we have expanded the exponential to first order in $(d / w)^{2}$, justified by the weakness of the first step of Stern-Gerlach separation.

Equation (8) is the starting point for the forthcoming discussion. First of all, we note that if the measurement is weak enough to satisfy

$$
\frac{d^{2}}{w^{2}} \ll \frac{\left|\alpha \gamma^{*}+\beta \delta^{*}\right|^{2}}{\left|\Re\left(\alpha \beta^{*} \gamma^{*} \delta\right)\right|}
$$

then the post-selected mean value reduces to

$$
\langle z\rangle_{\text {post }} \approx \Re\langle z\rangle_{\text {weak }}
$$

where

$$
\langle z\rangle_{\text {weak }}=\frac{\alpha \gamma^{*}-\beta \delta^{*}}{\alpha \gamma^{*}+\beta \delta^{*}} d=\frac{\left\langle f\left|\hat{\sigma}_{z} d\right| i\right\rangle}{\langle f \mid i\rangle}
$$

is just the Aharonov-Albert-Vaidman weak value (Eq. (11)) of the observable $\hat{A}=\hat{\sigma}_{z} d$.

Instead of focusing on the shift in coordinate, recent optical experiments [7, 8] measure the shift in transverse momentum $\hat{p}_{z}$, brought about by the phase difference introduced on post-selection. The corresponding mean 
value can be directly evaluated, to give

$$
\begin{aligned}
\left\langle p_{z}\right\rangle_{p o s t} & =\frac{\Im\left(\alpha \beta^{*} \gamma^{*} \delta\right) e^{-\frac{d^{2}}{2 w^{2}}}}{|\alpha|^{2}|\gamma|^{2}+|\beta|^{2}|\delta|^{2}+2 \Re\left(\alpha \beta^{*} \gamma^{*} \delta\right) e^{-\frac{d^{2}}{2 w^{2}}}} \frac{\hbar d}{w^{2}} \\
& \approx \frac{2 \Im\left(\alpha \beta^{*} \gamma^{*} \delta\right)(d / w)}{\left|\alpha \gamma^{*}+\beta \delta^{*}\right|^{2}-\Re\left(\alpha \beta^{*} \gamma^{*} \delta\right)(d / w)^{2}} w_{p},
\end{aligned}
$$

where $w_{p}=(\hbar / 2) / w$ is the width of the gaussian momentum distribution in the incoming beam. In the limiting case of Eq. (91), Eq. (12) reduces to

$$
\left\langle p_{z}\right\rangle_{\text {post }} \approx w_{p} \Im\langle z\rangle_{\text {weak }} \frac{d}{w} .
$$

Satisfying the condition formulated in Eq. (9) is far from being trivial though: on approaching post-selection into a spin state orthogonal to the preselected one, viz.

$$
\gamma \approx i \beta^{*} ; \quad \delta \approx-i \alpha^{*},
$$

which may look advantageous in enhancing the signal amplification factor, the numerator of the r.h.s. in the inequality (9) approaches zero. Then the $(d / w)^{2}$ terms in the denominators of Eqs. (8) and (12) are no more negligible, and it is getting necessary to use the full derivativeLorentzian shape for data fitting.

The rest of this note addresses that task. Deviations from the limiting case of Eq. (14) are explored in the following parametrization: we introduce the amplitude detuning $\epsilon$ through

$$
|\gamma|^{2}=|\beta|^{2}+\epsilon ; \quad|\delta|^{2}=|\alpha|^{2}-\epsilon
$$

as well as the phase detuning [13]

$$
\Delta=\left(\pi-\varphi_{\alpha}+\varphi_{\beta}+\varphi_{\gamma}-\varphi_{\delta}\right) / 2
$$

where e.g. $\varphi_{\alpha}$ is the phase angle of the complex amplitude $\alpha$. Both $\epsilon$ and $\Delta$ are under control of the experimenter, fixed for a given run; $d / w$ is the output. Expanding the full expressions, Eqs. (8) and (12) to leading powers in $\epsilon$ and $\Delta$, we obtain the final results

$$
\begin{aligned}
\langle z\rangle_{\text {post }} & =w \frac{2 \epsilon}{2|\alpha|^{2}|\beta|^{2}+\epsilon\left(|\alpha|^{2}-|\beta|^{2}\right)} \\
& \cdot \frac{d / w}{4 \Delta^{2}+\left(|\alpha|^{-4}+|\beta|^{-4}\right)\left(\epsilon^{2} / 8\right)+(d / w)^{2}} ;
\end{aligned}
$$

$$
\begin{aligned}
\left\langle p_{z}\right\rangle_{\text {post }} & = \\
& w_{p} \cdot \frac{2 \Delta(d / w)}{4 \Delta^{2}+\left(|\alpha|^{-4}+|\beta|^{-4}\right)\left(\epsilon^{2} / 8\right)+(d / w)^{2}} .
\end{aligned}
$$

From the above results we see that the fully linear amplification of the weak Stern-Gerlach displacement $d$ into the AAV-postselected mean value, be it real or imaginary, is violated as soon as the relative displacement $\eta=d / w$ reaches the order of magnitude of

$$
\eta_{0}=\left[4 \Delta^{2}+\left(|\alpha|^{-4}+|\beta|^{-4}\right)\left(\epsilon^{2} / 8\right)\right]^{1 / 2} .
$$

Actually, viewed as a function of $\eta$, the value $\eta=\eta_{0}$ corresponds to the maximum of post-selected deflection. Confirming what was expected from the phase contrast context, that maximum deflection is limited by the transverse width of the incoming beam, either in coordinate or in momentum.

For a given value of the weak displacement $d$ to be measured, the AAV limit is obtained for detunings $\Delta$ and/or $\epsilon$ large enough to assure $\eta_{0} \gg d / w$; otherwise the full derivative-Lorentzian form of Eqs. (17) and (18) should be used for data fitting, allowing one to reach higher levels of signal amplification. It may prove advantageous to build in a controlled value of $\epsilon \neq 0$, to eliminate errors from unwanted post-selection amplitude asymmetry.

The results presented above refer to gaussian beams. As noticed by Hosten and Kwiat [7], switching to momentum space is sensitive to details of the beam profile; however, the same can be true about the whole calculation [14]. In the one-dimensional case, adding some non-gaussian correction terms from a cumulant expansion would be straightforward; for real beams of twodimensional cross-section the procedure would be more complex. As a general trend, if measured mean values are calibrated to the zero deflection case, the remaining dominant corrections would appear in the overlap integrals replacing the last term in Eq. (6), the consequence being a shift of the turning point $\eta_{0}$ from the value given by Eq. (19).

We conclude the analysis by noting that the whole AAV procedure can be regarded as a late descendent of phase-contrast microscopy [15]. In both cases two overlapping components of slightly different space-dependent amplitudes are forced to exhibit a visible interference pattern, by introducing a constant phase difference between them [16], thereby turning potentially invisible coherence into large-scale visible interference.

The quantum case is complicated by the fact that the two overlapping beams are entangled to orthogonal spin components which makes their coherence strictly latent for straightforward interference counting 17]. Postselecting a spin component corresponding to a different space direction on the finite-dimension basis of spin states is an ingenious tool to introduce a phase difference and thereby creating visible phase contrast [18]. The light polarization case is analogous, although more classical in context: even light waves of orthogonal linear polarizations do interfere to produce circularly polarized waves; however, that interference remains invisible until analyzed (postselected) according to circular polarization. Rotating the basis of some other internal degree of freedom can be used to the same end; more sophisticated schemes are possible too, like that of two-photon entanglement [4]. The tool of spin basis rotation followed by selecting one of the components is by no means limited to the context of weak measurements; it is used for the preparation of so-called Schrödinger cat states in mesoscopic systems, which is an important step towards quantum information processing [19, 20]. A more detailed 
overview of the fascinating field of quantum phase contrast physics will be presented elsewhere [21].

A last issue of principle should be mentioned here. There is a growing culture of theories of weak measurements in a slightly different sense: accomplished but unsharp measurement of a "pointer variable" coupled to $\hat{A}$ (in our case, the pointer variable is $z$ ); in a certain limit, that unsharp measurement is carried out continuously in time [22, 23]. In the AAV context, a single act of weak measurement in that sense can be followed by quantum post-selection according to sharp measurement of a different pointer variable (here: $\xi$ ), the result being used to retain or reject data collected during the first step [23]. A promising implementation is the case of a double-quantum-dot qubit, on which the binary position of the electron is measured by means of a quantum point contact detector, first weakly, then in a strongly post-selecting way [24]. The analysis furnishes formu- las similar to ours; however, the relationship of the two problems to each other may need further clarification.

To summarize, the present note offers a clear and in the same time practical framework to discuss pre- and postselected weak measurements. As a first step in utilizing the advantages of the proposed approach, we have started exploring the parameter range next to orthogonal postselection, far beyond the applicability of the AharonovAlbert-Vaidman weak value, which is both accessible and important for signal amplification type experiments.

This work was partially supported by the Hungarian Scientific Research Fund OTKA under Grant No. T75129. The author is indebted to Lajos Diósi for enlightening discussions, and to J. Zsolt Bernád, Lev Vaidman, Andrew Jordan, Nathan Williams, and Alonso Botero for helpful comments on various drafts of the manuscript.
[1] Y. Aharonov, D.Z. Albert, and L. Vaidman, Phys. Rev. Lett. 60, 1351 (1988). For a recent detailed discussion, see the last three chapters in Y. Aharonov and D. Rohrlich, Quantum Paradoxes (Wiley-VCH, Weinheim 2005; a most recent brief review is that of S. Popescu, Physics 2, 32 (2009).

[2] M.V. Berry, in Quantum Coherence and Reality; in celebration of the 60th Birthday of Yakir Aharonov, J S Anandan and J L Safko, eds. (World Scientific, Singapore 1994), p. 55.

[3] N.W.M. Ritchie, J.G. Story, and R.G. Hulet, Phys. Rev. Lett. 66, 1107 (1991)

[4] G.J. Pryde, J.L. O'Brien, A.G. White, T.C. Ralph, and H.M. Wiseman, ibid. 94, 220405 (2005).

[5] One may find it somewhat strange that it is the two unitary steps which are usually called "Von Neumann measurements".

[6] Detectors being big, what we have in mind here is a detector with a narrow slit in front, scanning together along $z$, or a high-resolution detector array.

[7] O. Hosten and P. Kwiat, Science 319, 787 (2008).

[8] P.B. Dixon, D.J. Starling, A.N. Jordan, and J.C. Howell, Phys. Rev. Lett. 102, 173601 (2009).

[9] It is worth mentioning that signal amplification through post-selection is a genuine experimental advantage; to achieve the same enhancement of accuracy by more extensive data acquisition might require an unrealistic level of temporal stability of the measuring apparatus.

[10] More than a mathematical accident, there is a general algebraic reason behind: step Eq. (5) contains two complementary projections in the spin subspace, adding up to unity even if squared.

[11] That has been already noticed by Y. Aharonov and A. Botero, Phys. Rev. A 72, 052111 (2005), who carry on their analysis in terms of the interesting concept of "quantum averages of weak values".

[12] This also indicates that measurement of $z$, and postselection in $\xi$, are interchangeable (L. Diósi, private communication). The nontrivial point about it is that the relative phases carried by the spin amplitudes and ren- dered visible by post-selection, being the same for each $z$, are not lost on measuring $z$.

[13] With the factors $i$ in Eq. (14), our definition of $\Delta$ coincides with that of Hosten and Kwiat [7].

[14] L. Vaidman, private communication.

[15] F. Zernike, Physica 9, 686, 974 (1942).

[16] The most straightforward appearance of that idea in quantum mechanics is the tunneling current, induced by the Josephson phase difference between two oppositely oriented evanescent waves.

[17] The phrase "latent coherence" has been apparently first used in physics by D.M. Greenberger and A. YaSin, Found. Phys. 19, 679 (1988).

[18] Alternatively, that phase difference can be interpreted in terms of a geometrical phase; see S. Tamate, H. Kobayashi, T. Nakanishi, K. Sugiyama, and M. Kitano, New J. Phys. 11, 093025 (2009).

[19] B. Yurke and D. Stoler, Phys. Rev. Lett. 57, 13 (1986); C. Monroe, D.M. Meekhof, B.E. King, and D.J. Wineland, Science 272, 1131 (1996); M. Brune et al., Phys. Rev. Lett. 77, 4887 (1996).

[20] Another related emblematic post-selection protocol, the "quantum eraser", first proposed by M.O. Scully and K. Drühl, Phys. Rev. A 25, 2208 (1982), acts just the opposite way. To adapt the idea to the present case: destructive interference is first made latent by spin labeling, then recovered by projecting spins onto uniform, i.e. non-labeling basis states.

[21] T. Geszti, in preparation.

[22] For a recent review, see K. Jacobs and D.A. Steck, Contemp. Phys. 47, 279 (2006).

[23] The combination of unsharp quantum measurement and sharp post-selection is analyzed in detail by L. Diósi, in Encyclopedia of Mathematical Physics, edited by J.-P. Françoise, G.L. Naber, and S.T. Tsou (Elsevier, Oxford 2006), vol. 4, p. 276.

[24] N.S. Williams and A.N. Jordan, Phys. Rev. Lett. 100, 026804 (2008); A. Romito, Y. Gefen, and Y.M. Blanter, ibid. 100, 056801 (2008). 